\title{
HANKEL DETERMINANT FOR CERTAIN CLASS OF ANALYTIC FUNCTION DEFINED BY GEBERALIZED DERIVATIVE OPERATOR
}

\author{
MA`MOUN HARAYZEH AL-ABBADI AND MASLINA DARUS
}

\begin{abstract}
The authors in [1] have recently introduced a new generalised derivatives operator $\mu_{\lambda_{1}, \lambda_{2}}^{n, m}$, which generalised many well-known operators studied earlier by many different authors. By making use of the generalised derivative operator $\mu_{\lambda_{1}, \lambda_{2}}^{n, m}$, the authors derive the class of function denoted by $\mathscr{H}_{\lambda_{1}, \lambda_{2}}^{n, m}$, which contain normalised analytic univalent functions $f$ defined on the open unit disc $U=\{z \in \mathbb{C}:|z|<1\}$ and satisfy

$$
\operatorname{Re}\left(\mu_{\lambda_{1}, \lambda_{2}}^{n, m} f(z)\right)^{\prime}>0, \quad(z \in U) .
$$

This paper focuses on attaining sharp upper bound for the functional $\left|a_{2} a_{4}-a_{3}^{2}\right|$ for functions $f(z)=z+\sum_{k=2}^{\infty} a_{k} z^{k}$ belonging to the class $\mathscr{H}_{\lambda_{1}, \lambda_{2}}^{n, m}$.
\end{abstract}

\section{Introduction and Definitions}

Throughout this paper, we use the following notation

$$
\begin{aligned}
s & =\left[\left(1+\lambda_{2}\right)\left(1+3 \lambda_{2}\right)\right]^{m} \\
r & =\left[\left(1+\lambda_{1}\right)\left(1+3 \lambda_{1}\right)\right]^{m-1} \\
l & =\left(1+2 \lambda_{2}\right)^{2 m} \\
w & =\left(1+2 \lambda_{1}\right)^{2 m-2} .
\end{aligned}
$$

Let $\mathscr{A}$ denote the class of functions $f$ of the form

$$
f(z)=\sum_{k=0}^{\infty} a_{k} z^{k}, \quad a_{k} \quad \text { is complex number }
$$

which are normalised and analytic in the open unit disc $U=\{z \in \mathbb{C}:|z|<1\}$ on the complex plane $\mathbb{C}$. Consider $\mathscr{S}$ denote the subclass of $\mathscr{A}$ normalised analytic univalent functions $f$ of the form

$$
f(z)=z+\sum_{k=2}^{\infty} a_{k} z^{k}, \quad a_{k} \quad \text { is complex number }
$$

Corresponding author: Maslina Darus.

2010 Mathematics Subject Classification. 30C45.

Key words and phrases. Analytic function, univalent function, Fekete-Szegö functional, Hankel determinant, convex and starlike functions, positive real functions, derivative operator. 
Let $S^{*}(\alpha), K(\alpha)(0 \leq \alpha<1)$ denote the subclasses of $\mathscr{S}$ consisting of functions that are starlike of order $\alpha$ and convex of order $\alpha$ in $U$, respectively. In particular, the classes $S^{*}(0)=$ $S^{*}$ and $K(0)=K$ are the familiar classes of starlike and convex functions in $U$, respectively.

Let be given two functions $f(z)=z+\sum_{k=2}^{\infty} a_{k} z^{k}$ and $g(z)=z+\sum_{k=2}^{\infty} b_{k} z^{k}$ analytic in the open unit disc $U=\{z \in \mathbb{C}:|z|<1\}$. Then the Hadamard product (or convolution) $f * g$ of two functions $f, g$ is defined by

$$
f(z) * g(z)=(f * g)(z)=z+\sum_{k=2}^{\infty} a_{k} b_{k} z^{k} .
$$

Next, we state basic ideas on $(x)_{k}$, which denotes the Pochhammer symbol (or the shifted factorial) defined by

$$
(x)_{k}=\frac{\Gamma(x+k)}{\Gamma(x)}=\left\{\begin{array}{l}
1 \quad \text { for } k=0, x \in \mathbb{C} \backslash\{0\}, \\
x(x+1)(x+2) \ldots(x+k-1) \text { for } k \in \mathbb{N}=\{1,2,3, \ldots\} \text { and } x \in \mathbb{C} .
\end{array}\right.
$$

We need the following definitions throughout our investigations.

Definition 1.1. (Noonan and Thomas [15]). For the function $f$ given by (1.1) for $q \geq 1$ and $k \geq 0$, the $q^{\text {th }}$ Hankel determinant of $f$ is defined by

$$
H_{q}(k)=\left|\begin{array}{cccc}
a_{k} & a_{k+1} & \ldots & a_{k+q+1} \\
a_{k+1} & a_{k+2} & \ldots & a_{k+q+2} \\
\vdots & \vdots & \vdots & \vdots \\
a_{k+q-1} & a_{k+q} & \cdots & a_{k+2 q-2}
\end{array}\right| .
$$

This determinant has also been considered by several authors. For example Noor in [16] determined the rate of growth $H_{q}(k)$ as $k \rightarrow \infty$ for functions $f$ given by (1.2) with bounded boundary. Ehrenborg in [6] studied the Hankel determinant of exponential polynomials. The Hankel transform of an integer sequence and some of its properties were discussed by Layman in [11].

A classical theorem of Fekete and Szegö functional [7] considered the Hankel determinant of $f \in \mathscr{S}$ for $q=2$ and $n=1$,

$$
H_{2}(1)=\left|\begin{array}{ll}
a_{1} & a_{2} \\
a_{2} & a_{3}
\end{array}\right|
$$

They made an early study for the estimates of $\left|a_{3}-\mu a_{2}^{2}\right|$ when $a_{1}=1$ and $\mu$ real. The wellknown result due to this functional states that if $f \in \mathscr{S}$ then

$$
\left|a_{3}-\mu a_{2}^{2}\right| \leq \begin{cases}3-4 \mu, & \text { if } \mu \leq 0, \\ 1+2 \exp \left(\frac{-2 \mu}{1-\mu}\right), & \text { if } 0 \leq \mu \leq 1, \\ 4 \mu-3, & \text { if } \mu \geq 1 .\end{cases}
$$


Hummel [9, 8] proved the conjecture of Singh that $\left|a_{3}-a_{2}^{2}\right| \leq \frac{1}{3}$ for the class $\mathscr{C}$ of convex functions. Keogh and Merkes [10] obtained sharp estimates for $\left|a_{3}-\mu a_{2}^{2}\right|$ when $f$ is closeto-convex, starlike and convex in $U$. Here, we consider the Hankel determinant of $f \in \mathscr{S}$ for $q=2$ and $n=2$,

$$
H_{2}(2)=\left|\begin{array}{ll}
a_{2} & a_{3} \\
a_{3} & a_{4}
\end{array}\right|
$$

In the present paper, we seek upper bound for the functional $\left|a_{2} a_{4}-a_{3}^{2}\right|$ for functions $f$ belonging to the class $\mathscr{H}_{\lambda_{1}, \lambda_{2}}^{n, m}$. The subclass $\mathscr{H}_{\lambda_{1}, \lambda_{2}}^{n, m}$ is defined as the following:

Definition 1.2. Let $f$ be given by (1.2). Then $f$ is said to be in the class $\mathscr{H}_{\lambda_{1}, \lambda_{2}}^{n, m}$ if it satisfies the inequality

$$
\operatorname{Re}\left(\mu_{\lambda_{1}, \lambda_{2}}^{n, m} f(z)\right)^{\prime}>0, \quad(z \in U),
$$

where $\mu_{\lambda_{1}, \lambda_{2}}^{n, m} f(z)$ denote the generalised derivative operator which was introduced by the authors [1] earlier. The generalised derivative operator is given as the following:

Definition 1.3. For $f \in \mathscr{A}$ the generalised derivative operator $\mu_{\lambda_{1}, \lambda_{2}}^{n, m}$ is defined by $\mu_{\lambda_{1}, \lambda_{2}}^{n, m}: \mathscr{A} \rightarrow$ $\mathscr{A}$

$$
\mu_{\lambda_{1}, \lambda_{2}}^{n, m} f(z)=z+\sum_{k=2}^{\infty} \frac{\left(1+\lambda_{1}(k-1)\right)^{m-1}}{\left(1+\lambda_{2}(k-1)\right)^{m}} c(n, k) a_{k} z^{k}, \quad(z \in U),
$$

where $n, m \in \mathbb{N}_{0}=\{0,1,2 \ldots\},. \lambda_{2} \geq \lambda_{1} \geq 0$ and $c(n, k)=\left(\begin{array}{c}n+k-1 \\ n\end{array}\right)=\frac{(n+1)_{k-1}}{(1)_{k-1}}$.

Special cases of this operator includes the Ruscheweyh derivative operator in the cases $\mu_{\lambda_{1}, 0}^{n, 1} \equiv \mu_{0,0}^{n, m} \equiv \mu_{0, \lambda_{2}}^{n, 0} \equiv R^{n}$ [18], the Salagean derivative operator $\mu_{1,0}^{0, m+1} \equiv S^{n}$ [19], the generalised Ruscheweyh derivative operator $\mu_{\lambda_{1}, 0}^{n, 2} \equiv R_{\lambda}^{n}$ [4], the generalised Salagean derivative operator introduced by Al-Oboudi $\mu_{\lambda_{1}, 0}^{0, m+1} \equiv S_{\beta}^{n}$ [2], and the generalised Al-Shaqsi and Darus derivative operator $\mu_{\lambda_{1}, 0}^{n, m+1} \equiv D_{\lambda, \beta}^{n}$ [3]. It is easily seen that $\mu_{\lambda_{1}, 0}^{0,1} f(z)=\mu_{0,0}^{0, m} f(z)=\mu_{0, \lambda_{2}}^{0,0} f(z)=$ $f(z)$ and $\mu_{\lambda_{1}, 0}^{1,1} f(z)=\mu_{0,0}^{1, m} f(z)=\mu_{0, \lambda_{2}}^{1,0} f(z)=z f^{\prime}(z)$ and also $\mu_{\lambda_{1}, 0}^{a-1,0} f(z)=\mu_{0,0}^{a-1, m} f(z)$ where $a=1,2,3, \ldots$.

The subclass $\mathscr{H}_{\lambda_{1}, 0}^{0,1}$ was studied systematically by MacGregor [14] who indeed referred to numerous earlier investigations involving functions whose derivative has a positive real part.

We first state some preliminary lemmas which shall be used in our proof.

\section{Preliminary Results}

To establish our results, we recall the following:

Let $P$ be the family of all functions $p$ analytic in $U$ for which $\operatorname{Re}(p(z))>0$ and be given by the power series

$$
p(z)=1+c_{1} z+c_{2} z^{2}+\ldots . \quad(z \in U) .
$$


Lemma 2.1. (Pommerenke [17]). If $p \in P$. Then the sharp estimate

$$
\left|c_{k}\right| \leq 2 \quad \text { for each } k \text {, }
$$

and

$$
\left|c_{2}-\frac{c_{1}^{2}}{2}\right| \leq 2-\frac{\left|c_{1}\right|^{2}}{2} .
$$

Lemma 2.2. (Libera and Zlotkiewicz [12, 13]). Let the function $p \in P$ be given by the powers series (2.1). Then

$$
2 c_{2}=c_{1}^{2}+x\left(4-c_{1}^{2}\right),
$$

for some $x,|x| \leq 1$, and

$$
4 c_{3}=c_{1}^{3}+2\left(4-c_{1}^{2}\right) c_{1} x-c_{1}\left(4-c_{1}^{2}\right) x^{2}+2\left(4-c_{1}^{2}\right)\left(1-|x|^{2}\right) z,
$$

for some value of $z,|z|<1$.

\section{Main Result}

Our main result is the following:

Theorem 3.3. Let the function $f$, given by (1.2) be in the class $\mathscr{H}_{\lambda_{1}, \lambda_{2}}^{n, m}$. Then

$$
\left|a_{2} a_{4}-a_{3}^{2}\right| \leq \frac{16\left(1+2 \lambda_{2}\right)^{2 m}}{9(n+1)^{2}(n+2)^{2}\left(1+2 \lambda_{1}\right)^{2 m-2}} .
$$

The result obtained is sharp.

Proof. We refer to the method by Libera and Zlotkiewicz [12, 13]. For $f \in \mathscr{P}_{\lambda_{1}, \lambda_{2}}^{n, m}$, it follows from (1.3) that $\exists p \in P$ such that

$$
\left(\mu_{\lambda_{1}, \lambda_{2}}^{n, m} f(z)\right)^{\prime}=p(z)=1+c_{1} z+c_{2} z^{2}+\ldots
$$

for some $(z \in U)$. From (3.1) computation and equating coefficients, we obtain

$$
\left.\begin{array}{l}
a_{2}=\frac{\left(1+\lambda_{2}\right)^{m} c_{1}}{2(n+1)\left(1+\lambda_{1}\right)^{m-1}} \\
a_{3}=\frac{2\left(1+2 \lambda_{2}\right)^{m} c_{2}}{3(n+1)(n+2)\left(1+2 \lambda_{1}\right)^{m-1}} \\
a_{4}=\frac{3\left(1+3 \lambda_{2}\right)^{m} c_{3}}{2(n+1)(n+2)(n+3)\left(1+3 \lambda_{1}\right)^{m-1}}
\end{array}\right\} .
$$


From (3.2), it can be easily established that

$$
\left|a_{2} a_{4}-a_{3}^{2}\right|=\frac{1}{(n+1)^{2}(n+2)}\left|\frac{3 s c_{1} c_{3}}{4 r(n+3)}-\frac{4 l c_{2}^{2}}{9 w(n+2)}\right| .
$$

Where $s, r, l, w$ as mentioned before.

Since the function $p(z)$ is the member of the class $P$ simultaneously, we assume without loss of generality that $c_{1}>0$. For convenience of notation, we take $c_{1}=c(c \in[0,2])$.

Using (2.3) along with (2.4), we get

$$
\begin{aligned}
\left|a_{2} a_{4}-a_{3}^{2}\right|= & \frac{1}{(n+1)^{2}(n+2)} \\
\times & \mid \frac{3 s c^{4}+6 s\left(4-c^{2}\right) c^{2} x-3 s c^{2}\left(4-c^{2}\right) x^{2}+6 s c\left(4-c^{2}\right)\left(1-|x|^{2}\right) z}{16 r(n+3)} \\
& -\frac{l c^{4}}{9 w(n+2)}-\frac{l x^{2}\left(4-c^{2}\right)^{2}}{9 w(n+2)}-\frac{2 l c^{2}\left(4-c^{2}\right) x}{9 w(n+2)} \mid, \\
= & \frac{1}{(n+1)^{2}(n+2)} \mid\left(\frac{27 s w(n+2)-16 r l(n+3)}{144 r w(n+2)(n+3)}\right) c^{4} \\
& +\left(\frac{27 s w(n+2)-16 r l(n+3)}{72 r w(n+2)(n+3)}\right) c^{2}\left(4-c^{2}\right) x \\
& -\left(4-c^{2}\right) x^{2}\left(\frac{[27 s w(n+2)-16 r l(n+3)] c^{2}+64 r l(n+3)}{144 r w(n+2)(n+3)}\right) \\
& +\frac{3 s c\left(4-c^{2}\right)\left(1-|x|^{2}\right) z}{8 r(n+3)} \mid .
\end{aligned}
$$

By triangle inequality we have

$$
\begin{aligned}
\left|a_{2} a_{4}-a_{3}^{2}\right| \leq & \frac{1}{(n+1)^{2}(n+2)}\left\{\frac{|27 s w(n+2)-16 r l(n+3)| c^{4}}{144 r w(n+2)(n+3)}+\frac{3 s c\left(4-c^{2}\right)}{8 r(n+3)}\right. \\
& +\frac{c^{2}\left(4-c^{2}\right) \rho|27 s w(n+2)-16 r l(n+3)|}{72 r w(n+2)(n+3)} \\
& \left.+\frac{\left(4-c^{2}\right) \rho^{2}(c-2)(27 s w(n+2) c-16 r l(n+3)(c+2))}{144 r w(n+2)(n+3)}\right\}, \\
= & F(\rho) .
\end{aligned}
$$

With $\rho=|x| \leq 1$. We assume that the upper bound for (3.4) attains at the interior point of $\rho \in[0,1]$ and $c \in[0,2]$, then

$$
\begin{aligned}
F^{\prime}(\rho)= & \frac{1}{(n+1)^{2}(n+2)}\left\{\frac{c^{2}\left(4-c^{2}\right)|27 s w(n+2)-16 r l(n+3)|}{72 r w(n+2)(n+3)}\right. \\
& \left.+\frac{\left(4-c^{2}\right) \rho(c-2)(27 s w(n+2) c-16 r l(n+3)(c+2))}{72 r w(n+2)(n+3)}\right\} .
\end{aligned}
$$


And with elementary calculus, we can show that $F^{\prime}(\rho)>0$ for $\rho>0$, provided that $c-2<0$ and $(27 s w(n+2) c-16 r l(n+3)(c+2))<0$.

Now, our goal is to prove the inequality

$$
[27 s w(n+2) c-16 r l(n+3)(c+2)]<0 .
$$

Now, (3.5) can be simplified to

$$
s w(27 n+54) c<r l(16 n+48)(c+2) .
$$

So (3.6) is true provided that our two inequalities

$$
(27 n+54) c<(16 n+48)(c+2),
$$

and

$$
s w<r l \text {, }
$$

are satisfied.

First, we need to show the inequality (3.7) holds, so from (3.7) we have

$$
11 n c+6 c<32 n+96,
$$

and immediately implies that

$$
n(32-11 c)+6(16-c)>0 .
$$

Thus inequality (3.7) is true.

Next, we want to show the inequality $s w<r l$ holds. This inequality reduces to

$$
\left[\frac{\left(1+\lambda_{2}\right)\left(1+3 \lambda_{2}\right)\left(1+2 \lambda_{1}\right)^{2}}{\left(1+\lambda_{1}\right)\left(1+3 \lambda_{1}\right)\left(1+2 \lambda_{2}\right)^{2}}\right]^{m} \frac{\left(1+\lambda_{1}\right)\left(1+3 \lambda_{1}\right)}{\left(1+2 \lambda_{1}\right)^{2}}<1 .
$$

From (3.9), we must show that the inequalities

$$
\frac{\left(1+\lambda_{1}\right)\left(1+3 \lambda_{1}\right)}{\left(1+2 \lambda_{1}\right)^{2}}<1,
$$

and

$$
\frac{\left(1+\lambda_{2}\right)\left(1+3 \lambda_{2}\right)\left(1+2 \lambda_{1}\right)^{2}}{\left(1+\lambda_{1}\right)\left(1+3 \lambda_{1}\right)\left(1+2 \lambda_{2}\right)^{2}}<1,
$$

are true.

Now, from (3.10) it is easy to see that

$$
1+4 \lambda_{1}+3 \lambda_{1}^{2}<1+4 \lambda_{1}+4 \lambda_{1}^{2}
$$


and obviously

$$
\lambda_{1}^{2}>0
$$

Hence the proof is done for particular inequality (3.10).

Next we need to prove the inequality (3.11) is true. So, by doing tedious calculations for (3.11), we shall get

$$
\left(1+4 \lambda_{2}+3 \lambda_{2}^{2}\right)\left(1+4 \lambda_{1}+4 \lambda_{1}^{2}\right)<\left(1+4 \lambda_{1}+3 \lambda_{1}^{2}\right)\left(1+4 \lambda_{2}+4 \lambda_{2}^{2}\right),
$$

and a straightforward calculation and some simplifications, we can conclude that

$$
\lambda_{1}^{2}-\lambda_{2}^{2}+4 \lambda_{1}^{2} \lambda_{2}-4 \lambda_{2}^{2} \lambda_{1}<0
$$

and therefore

$$
\left(\lambda_{1}-\lambda_{2}\right)\left(\lambda_{1}+\lambda_{2}+4 \lambda_{1} \lambda_{2}\right)<0 .
$$

Hence the proof for inequality (3.11) is complete.

Now after satisfying the inequalities (3.5) and $c-2<0$ we observed that $F^{\prime}(\rho)>0$ for $\rho \in[0,1]$, implying that $F$ is an increasing function and thus the upper bound for (3.4) corresponds to $\rho=1$ and so $\max F(\rho)=F(1)$. This contradicts our assumption of having the maximum value in the interior of $\rho \in[0,1]$.

Now let,

$$
\begin{aligned}
G(c)=F(1)= & \frac{1}{(n+1)^{2}(n+2)}\left\{\frac{|27 s w(n+2)-16 r l(n+3)| c^{4}}{144 r w(n+2)(n+3)}+\frac{3 s c\left(4-c^{2}\right)}{8 r(n+3)}\right. \\
& +\frac{c^{2}\left(4-c^{2}\right)|27 s w(n+2)-16 r l(n+3)|}{72 r w(n+2)(n+3)} \\
& \left.+\frac{\left(4-c^{2}\right)(c-2)(27 s w(n+2) c-16 r l(n+3)(c+2))}{144 r w(n+2)(n+3)}\right\} .
\end{aligned}
$$

Assume that $G(c)$ has a maximum value in an interior of $c \in[0,2]$, by elementary calculation we find

$$
\begin{aligned}
G^{\prime}(c) & =\frac{c}{36 r w(n+1)^{2}(n+2)^{2}(n+3)}\left[\left(4-c^{2}\right)|27 s w(n+2)-16 r l(n+3)|\right. \\
& \left.+27 s w(n+2)\left(2-c^{2}\right)+16 r l(n+3)\left(c^{2}-4\right)\right] .
\end{aligned}
$$

Then $G^{\prime}(0)=0$ implies the real critical point $c_{\bullet}=0$ or

$$
c_{*}=\sqrt{\frac{64 r l(n+3)-54 s w(n+2)-4|27 s w(n+2)-16 r l(n+3)|}{16 r l(n+3)-27 s w(n+2)-|27 s w(n+2)-16 r l(n+3)|}} .
$$


Through some calculations we observe that $c_{*}>2$, however $c_{*}$ is out of the interval $[0,2]$. A calculation showed that the maximum value occurs at $c=0$ or $c=c_{*}$ which contradicts our assumption of having the maximum value at the interior point of $c \in[0,2]$. Thus any maximum point of $G$ must be on the boundary of $c \in[0,2]$.

At $c=0$, we have

$$
G(c)=G(0)=\frac{16 l}{9 w(n+2)},
$$

and at $c=2$, we obtain

$$
G(c)=G(2)=\frac{|27 s w(n+2)-16 r l(n+3)|}{9 r w(n+2)(n+3)} .
$$

It is obvious that $G(0)>G(2)$ for the two choices of $|27 s w(n+2)-16 r l(n+3)|$. Hence $G$ attains maximum value at $c=0$. Therefore the upper bound for (3.4) corresponds to $\rho=1$ and $c=0$ in which case

$$
\left|a_{2} a_{4}-a_{3}^{2}\right| \leq \frac{16\left(1+2 \lambda_{2}\right)^{2 m}}{9(n+1)^{2}(n+2)^{2}\left(1+2 \lambda_{1}\right)^{2 m-2}} .
$$

Equality holds for the functions in $\mathscr{H}_{\lambda_{1}, \lambda_{2}}^{n, m}$ given by

$$
f^{\prime}(z)=\frac{1+z^{2}}{1-z^{2}}
$$

This concludes the proof of our theorem.

Note that this problem has yet to be solved for certain classes introduced in various studies (see for examples [5], [21], [22] and [23]). Note that Hankel problems have also been solved successfully for fractional operator which can be seen in [20].

\section{Acknowledgement}

This work is fully supported by UKM-ST-06-FRGS0244-2010, Malaysia.

\section{References}

[1] M. H. Al-Abbadi and M. Darus, Differential subordination for new generalised derivative operator, Acta. Univ. Apul., 20(2009), 265-280.

[2] F. M. Al-Oboudi, On univalent functions defined by a generalised Salagean Operator, Int. J. Math. Math. Sci., 27(2004), 1429-1436.

[3] K. Al-Shaqsi and M. Darus, Differential Subordination with generalised derivative operator, Int. J. Comp. Math. Sci, 2(2), (2008), 75-78.

[4] K. Al-Shaqsi and M. Darus, On univalent functions with respect to $k$-symmetric points defined by a generalised Ruscheweyh derivative operator, Journal of Analysis and Application, 7(1), (2009), 53-61.

[5] K. Al-Shaqsi, M. Darus and O. A. Fadipe-Joseph, A new subclass of Salagean-type harmonic univalent functions, Abstract and Applied Analysis, vol. 2010 (2010), Article ID 821531, 12 pages. doi:10.1155/2010/821531. 
[6] R. Ehrenborg, The Hankel determinant of exponential polynomials, American Mathematical Monthly, 107(2000), 557-560.

[7] M. Fekete and G. Szegö, Eine bemerkung über ungerade schlichte functionen, J. Lond. Math. Soc., 8(1933), 85-89.

[8] J. Hummel, Extremal problems in the class of starlike functions, Proc. Amer. Math. Soc., 11(1960), 741-749.

[9] J. Hummel, The coefficient regions of starlike functions, Pacific J. Math., 7(1957), 1381-1389.

[10] F. R. Keogh and E. P. Merkes, A coefficient inequality for certain classes of analytic functions, Proc. Amer. Math. Soc., 20 (1969), 8-12.

[11] J. W. Layman, The Hankel transform and some of its properties, J, Integer Sequences, 4(2001), 1-11.

[12] R. J. Libera and E. J. Zlotkiewicz, Coefficient bounds for the inverse of a function with derivative in P, Proc. Amer. Math. Soc., 87(2) (1983), 251-289.

[13] R. J. Libera and E.J. Zlotkiewicz, Early coefficients of the inverse of a regular convex function, Proc. Amer. Math. Soc., 85(2) (1982), 225-230.

[14] T. H. Macgregor, Functions whose derivative has a positive real part, Trans. Amer. Math. Soc., 104 (1962), 532-537.

[15] J. W. Noonan and D. K. Thomas, On the second Hankel determinant of a really mean p-valent functions, Trans. Amer. Math. Soc, 223(2) (1976), 337-346

[16] K. I. Noor, Hankel determinant problem for the class of functions with bounded boundary rotation, Rev. Roum. Math. Pures Et Appl., 28(8) (1983), 731-739.

[17] CH. Pommerenke, Univalent functions, Vandenhoeck and Ruprecht, Göttingen, 1975.

[18] St. Ruscheweyh, New criteria for univalent functions, Proc. Amer. Math. Soc., 49 (1975), 109-115.

[19] G. S. Salagean, Subclasses of univalent functions, Lecture Notes in Math. (Springer-Verlag), 1013(1983), 362372.

[20] O. Al-Refai and M. Darus, Second Hankel determinant for a class of analytic functions defined by a fractional operator, European Journal of Scientific Research, 28(2) (2009), 234-241.

[21] F. Al-Kasasbeh and M. Darus, New subclass of analytic functions with some applications, European Journal of Scientific Research, 28(1) (2009), 124-131.

[22] A. Mohammed, M.Darus and D. Breaz, Fractional calculus for certain integral operator involving logarithmic coefficients, J. Math. Stat., 5(2) (2009), 118-122.

[23] R. W. Ibrahim and M. Darus, Operator defined by convolution with Zeta functions, Far East J. Math Sci., 40(1) (2010), 93-105.

School of Mathematical Sciences, Faculty of Science and Technology, Universiti Kebangsaan Malaysia, Bangi 43600 Selangor D. Ehsan, Malaysia.

E-mail: mamoun_nn@yahoo.com

School of Mathematical Sciences, Faculty of Science and Technology, Universiti Kebangsaan Malaysia, Bangi 43600 Selangor D. Ehsan, Malaysia.

E-mail: maslina@ukm.my 